doi:10.13108/2013-5-3-11

\title{
COMMUTING DIFFERENTIAL OPERATORS OF ORDER 4 AND 6
}

\author{
F.KH. BAICHOROVA, Z.S. ELKANOVA
}

\begin{abstract}
We consider a model problem on a pair of commuting differential operators of order 4 and 6 . The results are employed to generalize a known commuting pair in a work of J. Dixmier for the case of rational coefficients.
\end{abstract}

Keywords: commuting differential operators, differential operators of order 4 and 6 .

Mathematics Subject Classification: 34L05.

\section{INTRODUCTION}

Reformulating a question from the work by Burchnall and Chaundy [1] 1932, we arrive at the problem on a pair of polynomials $a(D)$ and $b(D)$ with constant coefficients satisfying the functional equation [7]

$$
a(D+\beta) b(D)=a(D) b(D+\alpha) ; \quad \alpha, \beta \in \mathbb{C}^{N}
$$

Here $D=\left(D_{1}, D_{2}, \ldots, D_{N}\right)$ is the formal variable and vectors $\alpha$ and $\beta$ in $\mathbb{C}^{N}$ are assumed to be given. For a differential operator $C(D)$ with partial variables $D_{j}=\partial j$, the formula

$$
C(D) \circ e^{\gamma \cdot x}=e^{\gamma \cdot x} C(D+\gamma), \quad \gamma \in \mathbb{C}^{N}, \quad C(D) \text { is a polynomial, }
$$

holds true. It follows from this formula that functional equation (1) is equivalent to commuting of a pair of partial differential operators semi-invariant w.r.t. the group of shifts,

$$
A=e^{\alpha \cdot x} \cdot a(D), \quad B=e^{\beta \cdot x} \cdot b(D) .
$$

Indeed, by (2), the composition of these operators leads us to the formula

$$
A \circ B=e^{(\alpha+\beta) \cdot x} a(D+\beta) b(D),
$$

and thus, the condition of commuting for such operators (3) is indeed reduced to (1).

In the theory of commutative rings of differential operators with one independent variables, special operators like $(3)^{1}$ can play the role of a model (cf. [6] and [8]). In the one-dimensional case, polynomial equation (1) casts into the form

$$
a(z+\beta) b(z)=b(z+\alpha) a(z), \quad \alpha, \beta \in C, \quad \alpha \beta \neq 0 .
$$

By means of dilatation $z$ with a coefficient $\beta_{n}$, it can be rewritten as

$$
a(z+n) b(z)=a(z) b(z+m), \quad m=\operatorname{deg} P(z), n=\operatorname{deg} Q(z) .
$$

Here there is no loss of generality. Indeed, let polynomials

$$
a(z)=z^{m}+a_{1} z^{m-1}+\cdots+a_{m}, \quad b(z)=z^{n}+b_{1} z^{n-1}+\cdots+b_{n}
$$

F.Kh. Baichorova, Z.S. Elkanova, Commuting differential operators of order 4 and 6.

(C) Baichorova F.Kh., Elkanova Z.S. 2013.

Submitted May 16, 2013.

${ }^{1}$ their eigenfunctions generalize Bessel functions 
satisfy functional equation (4). Equating the coefficients at $z^{n+m-1}$ and $z^{n+m-2}$ in the left hand side and the right hand side of the equation, we obtain

$$
\frac{\beta}{\alpha}=\frac{n}{m}, \quad b_{1}=\frac{n}{m} a_{1}+\frac{n}{2}(\beta-\alpha) .
$$

After the dilatation $z$, the former of these relations allows us to let $\alpha=m, \beta=n$, while the other expresses $b_{1}$ in terms of $a_{1}$. Continuing this process of equating like terms in equation (5), we can express all the coefficients $b_{1}, \ldots, b_{n}$ in terms of $a_{1}, \ldots, a_{m}$. Substituting these formulas for the coefficients of polynomial $b(z)$ into equation (5), we obtain that the degree of the polynomial

$$
c(z)=a(z+n) b(z)-a(z) b(z+m)
$$

satisfies the inequality

$$
\operatorname{deg} c(z) \leqslant m-2 \text {. }
$$

The remaining coefficients at $z^{j}, j \leqslant m-2$ give in this way $m-1$ equations for $m$ coefficients $a_{1}, \ldots, a_{m}$. By help of shift (2), we can let $a_{m}=0$ and equalize the number of equations and unknowns.

For coprime numbers $(m, n)$, the problem on commuting differential operators of orders $m$ and $n$ is studied rather well. In particular, in the considered case, for fixed $(m, n)$ and $\operatorname{gcd}(m, n)=1$ the complete lists of the polynomials satisfying equation (5) are given in work [6] (cf. also [8]). A feature of these polynomials is that their roots are integer as $a_{m}=0$. Besides this normalization conditions, it is taken into account in the lists that passing to adjoint operators does not break the commuting. At that, the formally adjoint operator for $\exp (\gamma \cdot x) \circ C(D)$ (see (2) ) reads as follows

$$
C(-D) \circ e^{\gamma \cdot x}=e^{\gamma \cdot x} C(\gamma-D) .
$$

Latterly an interest to a more complicated case $\operatorname{gcd}(m, n) \neq 1$ considerably increases. Basically, one considers commuting differential operators with polynomial coefficients generalizing the well-known Dixmier example [2] (the survey of appropriate references can be found in [4]).

In our model problem, equations (4) and (5) allow us to solve completely the issue on pairs of commuting operators of orders 4 and 6 . In particular, it is established that the operators

$$
A=e^{4 t} D^{2}(D+2)^{2}=A_{2}^{2}, \quad B=e^{6 t} D^{2}(D+2)^{2}(D+4)^{2}=A_{2}^{3}, \quad A_{2}=e^{2 t} D^{2}
$$

can serve as canonical forms. As one can see easily, their simultaneous eigenfunction $A_{2} \psi=\psi$ is the Bessel function of zero order that, as $n=0$, satisfies the equation

$$
y^{\prime \prime}+\frac{1}{x} y^{\prime}=\frac{x^{2}+n^{2}}{x^{2}} y, \quad D_{t}=-x D_{x}, x=-e^{-t} .
$$

For this model problem we succeeded to clarify an important role of additional 1 free parameter appearing in the commuting pairs of differential operators as $\operatorname{gcd}(m, n) \neq 1$.

\section{General properties of solutions to equation (5)}

Let us show that the polynomials $a(\lambda)$ and $b(\lambda)$ associated with commuting operators $A=$ $e^{m t} a(D)$ and $B=e^{n t} b(D)$ should have a simultaneous root $\alpha$. For the sake of simplicity, we shall assume that the roots of the polynomials are real (for the case of complex roots, the arguments are same).

The existence of simultaneous roots of the polynomials satisfying (5) is implied by

Lemma 1 (on simultaneous root). Suppose polynomials $a(\lambda)$ and $b(\lambda)$ of degrees $m$ and $n$ satisfy equation (5). Then these polynomials have a simultaneous root.

\footnotetext{
${ }^{1}$ not related with the spectral curve
} 
Proof. It was mentioned above that the shift does not break the commuting. By help of such shift we can assume that the roots of polynomial $a(\lambda)$ are non-positive and $a(0)=0$,

$$
a(\lambda)=\lambda \prod_{1}^{m-1}\left(\lambda+\lambda_{j}\right), \quad \lambda_{m-1} \leqslant \lambda_{m-2} \cdots \leqslant \lambda_{2} \leqslant \lambda_{1} \leqslant 0 .
$$

We shall show now that if polynomial $b(\lambda)$ satisfies $(5)$, then $b(0)=0$ and

$$
b(\lambda)=\lambda \prod_{1}^{n-1}\left(\lambda+\mu_{j}\right), \quad \mu_{n-1} \leqslant \mu_{n-2} \cdots \leqslant \mu_{2} \leqslant \mu_{1} \leqslant 0, \quad\left(\mu_{n-1}-\lambda_{m-1}\right)=n-m .
$$

We first assume that $b(0) \neq 0$. Then letting $\lambda=0$, in (5) we get

$$
\begin{gathered}
a(n) b(0)=a(0) b(m), \\
a(n) b(0)=0 \Rightarrow a(n)=0 .
\end{gathered}
$$

But $n>0$ that contradicts the absence of positive roots for polynomial $a(\lambda)$.

In the same way, assuming that polynomial $b(\lambda)$ has a positive root $\lambda_{0}$, by equation (5) we find

$$
\begin{gathered}
a\left(\lambda_{0}+n\right) b\left(\lambda_{0}\right)=a\left(\lambda_{0}\right) b\left(\lambda_{0}+m\right)=0, \\
a\left(\lambda_{0}\right) b\left(\lambda_{0}+m\right)=0 .
\end{gathered}
$$

But since $\lambda_{0}>0$ and by assumption $a(\lambda)$ has no positive roots, we have $b\left(\lambda_{0}+m\right)=0$. Repeating these arguments, we obtain an infinite series of zeroes $b(\lambda)$ which is impossible.

The latter of formulas 12 can be proven by passing to adjoint differential operators

$$
\begin{aligned}
A^{*} & =D\left(D-\lambda_{1}\right)\left(D-\lambda_{2}\right) \ldots\left(D-\lambda_{m-1}\right) e^{m t} \\
& =e^{m t}(D+m)\left(D-\lambda_{1}+m\right)\left(D-\lambda_{2}+m\right) \ldots\left(D-\lambda_{m-1}+m\right), \\
B^{*} & =D\left(D-\mu_{1}\right)\left(D-\mu_{2}\right) \ldots\left(D-\mu_{n-1}\right) e^{n t} \\
& =e^{n t}(D+n)\left(D-\mu_{1}+n\right)\left(D-\mu_{2}+n\right) \ldots\left(D-\mu_{n-1}+n\right) .
\end{aligned}
$$

We recall that if differential operators $A$ and $B$ commute, their adjoint operators $A^{*}$ and $B^{*}$ commute as well. Therefore, by the first part of the statement, their maximal roots should coincide. The maximal roots for adjoint operators $A^{*}$ and $B^{*}$ are respectively $\left(\lambda_{m-1}-m\right)$ and $\left(\mu_{n-1}-n\right)$. It implies the desired formula

$$
\lambda_{m-1}-m=\mu_{n-1}-n \Rightarrow\left(\mu_{n-1}-\lambda_{m-1}\right)=n-m .
$$

Generally speaking, the roots can be multiple, as the following example shows.

One can make sure that operators $A$ and $B$ of orders 4 and 6 ,

$$
\begin{gathered}
A=e^{4 t} D(D+2)(D+\alpha)(D+\alpha+2), \\
B=e^{6 t} D(D+2)(D+4)(D+\alpha)(D+\alpha+2)(D+\alpha+4),
\end{gathered}
$$

commute for each $\alpha$ and as $\alpha=2$, have multiple roots.

In the case $m$ and $n$ are coprime, in known to us cases there are multiple roots.

Lemma 2. Solutions of the polynomial equation

$$
P\left(z+n_{1}\right) Q(z)=P(z) Q\left(z+n_{2}\right)
$$

can be multiplied. 
Proof. Let $\left(p_{1}, q_{1}\right)$ and $\left(p_{2}, q_{2}\right)$ be two solutions of considered polynomial equation. Then $(p=$ $\left.p_{1} p_{2}, q=q_{1} q_{2}\right)$ is also a solution,

$$
p_{1}\left(\xi+n_{1}\right) q_{1}(\xi)=p_{1}(\xi) q_{1}\left(\xi+n_{2}\right), \quad p_{2}\left(\xi+n_{1}\right) q_{2}(\xi)=p_{2}(\xi) q_{2}\left(\xi+n_{2}\right)
$$

since

$$
p_{1}\left(\xi+n_{1}\right) p_{2}\left(\xi+n_{1}\right) q_{1}(\xi) q(\xi)=p_{1}(\xi) p_{2}(\xi) q_{1}\left(\xi+n_{2}\right) q_{2}\left(\xi+n_{2}\right)
$$

We observe that a dilatation of the independent variable in equation (5) gives

$$
P_{k}(z)=k^{m} P\left(\frac{z}{k}\right), Q_{k}(z)=k^{n} Q\left(\frac{z}{k}\right) \text { therefore, } \quad P_{k}(z+n k) Q_{k}(z)=P_{k}(z) Q_{k}(z+m k) .
$$

The coefficients $a_{j}(k)$ of polynomials $P_{k}(z)\left(Q_{k}(z)\right)$ are related with original ones by the formulas $a_{j}(k)=k^{j} a_{j}$.

Example 1. In the case of operators of second and third order, polynomials $P(z)$ and $Q(z)$ read as follows,

$$
P(z)=z^{2}+a_{1} z+a_{2}, \quad Q(z)=z^{3}+b_{1} z^{2}+b_{2} z+b_{3} .
$$

Employing Lemma 1, we represent these polynomials as

$$
P(z)=z(z+a), \quad Q(z)=z(z+b)(z+a+1), \quad 0 \leqslant b \leqslant a+1,0 \leqslant a .
$$

Equation (5) is as follows,

$(z+3)\left[z^{2}+(a+b+1) z+b+a b\right]=(z+2)\left[z^{2}+(a+b+2) z+2 a+a b\right], \quad$ therefore, $\quad a=1,3$.

Thus, we obtain two pairs of commuting differential operators of second and third order,

$$
P(z)=z^{2}+z=z(z+1), \quad Q(z)=z^{3}+3 z^{2}+2 z=z(z+1)(z+2)
$$

and

$$
P(z)=z^{2}+3 z=z(z+3), \quad Q(z)=z^{3}+6 z^{2}+8 z=z(z+2)(z+4) .
$$

2. Polynomial equation (5) as $m=4, n=6$

Taking into consideration Lemma 2, let us consider in greater details the polynomial equation

$$
P(z+3) Q(z)=P(z) Q(z+2),
$$

not fixing in advance the degrees of polynomials $P(z)$ and $Q(z)$. The technique developed in [6] allows one to study the commuting pairs of the operators whose orders are not coprime. The only difference is appearance of free parameters in the coefficients of polynomials $A(D)$ and $B(D)$ satisfying equation (16).

We return to operators of order 2-3 (cf. Example 1). In order to find polynomials $P(z)$ and $Q(z)$ of second and third order,

$$
P(z)=z^{2}+a_{1} z+a_{2}, \quad Q(z)=z^{3}+b_{1} z^{2}+b_{2} z+b_{3},
$$

satisfying equation (16), we equate coefficients at the like powers of $z$ in the left hand side and right hand side of these equation. Letting $a_{2}=0$, we find,

$$
b_{1}=\frac{3}{2} a_{1}+\frac{3}{2}, \quad b_{2}=\frac{3}{8} a_{1}^{2}+\frac{3}{2} a_{1}+\frac{1}{8}, \quad b_{3}=-\frac{1}{16} a_{1}^{3}+\frac{3}{16} a_{1}^{2}+\frac{1}{16} a_{1}-\frac{3}{16}=0
$$

and obtain $a_{1}=1,-1,3$ solving equation $b_{3}=0$. As $a_{1}=1$, we find $b_{1}=3, b_{2}=2, b_{3}=0$. And the pair of commuting polynomials read as

$$
P(z)=z^{2}+z=z(z+1), Q(z)=z^{3}+3 z^{2}+2 z=z(z+1)(z+2) .
$$


As $a_{1}=3$, we have $b_{1}=6, b_{2}=8, b_{3}=0$, and the pair of commuting polynomials is

$$
P(z)=z^{2}+3 z=z(z+3), Q(z)=z^{3}+6 z^{2}+8 z=z(z+2)(z+4) .
$$

As $a_{1}=-1$, we have $b_{1}=0, b_{2}=-1, b_{3}=0$, and the pair of commuting polynomials reads as

$$
P(z)=z^{2}-z=z(z-1), \quad Q(z)=z^{3}-z=z(z-1)(z+1) .
$$

In the case of operators of forth and sixth order, the commuting polynomials are

$$
\left\{\begin{array}{l}
P(z)=z^{4}+a_{1} z^{3}+a_{2} z^{2}+a_{3} z+a_{4}, \quad P(z+3)=z^{4}+p_{1} z^{3}+p_{2} z^{2}+p_{3} z+p_{4} \\
Q(z)=z^{6}+b_{1} z^{5}+b_{2} z^{4}+b_{3} z^{3}+b_{4} z^{2}+b_{5} z+b_{6}, Q(z+2)=z^{6}+\sum_{j=1}^{6} q_{j} z^{6-j} .
\end{array}\right.
$$

Taylor formula yields

$$
\begin{gathered}
p_{1}=\frac{1}{6} P^{\prime \prime \prime}(3)=a_{1}+12, p_{2}=a_{2}+9 a_{1}+54, p_{3}=a_{3}+6 a_{2}+27 a_{1}+12 \cdot 9, p_{4}=P(3) \\
q_{1}=b_{1}+12, q_{2}=b_{2}+10 b_{1}+60, q_{3}=b_{3}+8 b_{2}+40 b_{1}+160, \\
q_{4}=b_{4}+6 b_{3}+24 b_{2}+80 b_{1}+15 \cdot 16, q_{5}=b_{5}+4 b_{4}+12 b_{3}+32 b_{2}+80 b_{1}+192, q_{6}=Q(2) .
\end{gathered}
$$

The criterion for commuting of associated operators is reduced to polynomial equation (16) by dilatation of (15) with $k=2$.

Lemma 3. Multiplication of the solutions to equation (5) for operators of second and third order leads one, up to adjoint (i.e., Darboux transformation of zero order [8]), to the following list of commuting pairs of operators of order 4 and 6 ,

$$
\begin{array}{ll}
e^{4 t} D(D+2)(D+\alpha)(D+\alpha+2), & \left.e^{6 t} D(D+2)(D+4)(D+\alpha)(D+\alpha+2)(D+\alpha+4) \mathrm{A}_{1}\right) \\
e^{4 t} D(D+2)(D+\alpha)(D+\alpha+6), & \left.e^{6 t} D(D+2)(D+4)(D+\alpha)(D+\alpha+4)(D+\alpha+8) \mathrm{A}_{2}\right) \\
e^{4 t} D(D+6)(D+\alpha)(D+\alpha+6), & \left.e^{6 t} D(D+4)(D+8)(D+\alpha)(D+\alpha+4)(D+\alpha+8) \mathrm{A}_{3}\right)
\end{array}
$$

This list can be supplemented by the trivial pair of commuting differential operators

$$
e^{4 t} D(D+1)(D+2)(D+3), \quad e^{6 t} D(D+1)(D+2)(D+3)(D+4)(D+5)
$$

Proof. Choosing $k=2$ in equation (15), by known commuting operators of second and third order we obtain commuting operators of order 4 and 6 ,

$$
\begin{aligned}
& A_{1}=e^{2 t} D(D+1), B_{1}=e^{3 t} D(D+1)(D+2), \\
& A_{2}=e^{2 t} D(D+3), B_{2}=e^{3 t} D(D+2)(D+4) .
\end{aligned}
$$

According to Example 1, this list reads as follows,

$$
P_{1}(\xi)=\xi(\xi+1), Q_{1}(\xi)=\xi(\xi+1)(\xi+2), \quad P_{2}(\xi)=\xi(\xi+3), Q_{2}(\xi)=\xi(\xi+2)(\xi+4) .
$$

We can write

$$
\begin{gathered}
P=P_{1}^{2}=z^{2}(z+2)^{2}, Q=Q_{1}^{2}=z^{2}(z+2)^{2}(z+4)^{2} \\
P=P_{2}^{2}=z^{2}(z+6)^{2}, Q=Q_{2}^{2}=z^{2}(z+4)^{2}(z+8)^{2} \\
P=P_{1} P_{2}=z^{2}(z+2)(z+6), Q=Q_{1} Q_{2}=z^{2}(z+2)(z+4)^{2}(z+8) .
\end{gathered}
$$

Taking into consideration that the shift of the root does not break the commuting of the operators, up to adjoint, we obtain the desired list of the operators of order 4 and 6 . 
It can be shown that odd $\alpha$ (and even $\alpha$ ) lead us respectively to half-integer and integer $n$ in Bessel equation (10).

Remark. Solutions (17) of polynomial equation (16) normalized by the conditions $a_{4}=$ $b_{6}=0$ depend on the additional parameter $t=a_{1}$. At that, $\operatorname{deg} P_{2}=2, \operatorname{deg} Q_{3}=3$ and polynomial equation

$$
P_{2}(z+3) Q_{3}(z)=P_{2}(z) Q_{3}(z+2)
$$

holds true.

Equating coefficients at like powers of $z$, we express first all the coefficients $b_{i}$ in terms of $a_{1}, a_{2}, a_{3}$ (by help of shift, we vanish coefficient $a_{4}$ ),

$$
\begin{gathered}
b_{6} p_{4}=0, \quad b_{6} p_{3}+b_{5} p_{4}=a_{3} q_{6}, \quad b_{6} p_{2}+b_{5} p_{3}+b_{4} p_{4}=a_{2} q_{6}+a_{3} q_{5}, \\
p_{2}+b_{1} p_{1}+b_{2}=q_{2}+q_{1} a_{1}+a_{2}, \quad p_{3}+b_{1} p_{2}+b_{2} p_{1}=q_{3}+q_{2} a_{1}+q_{1} a_{2}+a_{3} .
\end{gathered}
$$

At $z^{10}$ and $z^{9}$, the identity holds immediately. Then we find

$$
\begin{aligned}
2 b_{1}= & 3 a_{1}+6, \quad 4 b_{2}=6 a_{2}+10+15 a_{1}+\frac{3}{2} a_{1}^{2}, \\
16 b_{3}= & 48 a_{2}+40 a_{1}+12 a_{1}^{2}+24 a_{3}+12 a_{2} a_{1}-a_{1}^{3}, \\
32 b_{4}= & \frac{3}{4} a_{1}^{4}-4+24 a_{3} a_{1}-3 a_{1}^{3}+72 a_{2}+72 a_{3}+3 a_{1}^{2}+36 a_{2} a_{1}-6 a_{2} a_{1}^{2}+12 a_{2}^{2}, \\
32 b_{5}= & 24 a_{2}-6 a_{1}^{2} a_{3}+3 a_{2} a_{1}^{3}-6 a_{1} a_{2}^{2}+24 a_{3} a_{2}+\frac{7}{2} a_{1}^{3}-\frac{3}{8} a_{1}^{5}-2 a_{1}+12 \\
& +96 a_{3}-9 a_{1}^{2}-12 a_{2} a_{1}+24 a_{1} a_{3}-6 a_{2} a_{1}^{2}+\frac{3}{4} a_{1}^{4}+12 a_{2}^{2}, \\
64 b_{6}= & \frac{7}{16} a_{1}^{6}-36-6 a_{1}^{2} a_{3}+3 a_{2} a_{1}^{3}-6 a_{1} a_{2}^{2}+24 a_{3} a_{2}+\frac{9}{2} a_{1}^{3}--\frac{15}{4} a_{1}^{4} a_{2}-\frac{3}{8} a_{1}^{5} \\
& +6 a_{1}-76 a_{2}+72 a_{3}-24 a_{3} a_{2} a_{1}+28 a_{1}^{2}+9 a_{2}^{2} a_{1}^{2}-24 a_{2} a_{1}+24 a_{3}^{2} \\
& +6 a_{3} a_{1}^{3}-4 a_{2}^{3}--28 a_{1} a_{3}+37 a_{2} a_{1}^{2}-\frac{13}{2} a_{1}^{4}-44 a_{2}^{2} .
\end{aligned}
$$

Due to Lemma 1 , we can let $b_{6} \stackrel{\text { def }}{=} \rho\left(a_{1}, a_{2}, a_{3}\right)=0$. At that,

$$
R(z)=P(z+3) Q(z)-P(z) Q(z+2) \Rightarrow R(0)=0, \quad R(z)=z r\left(a_{1}, a_{2}, a_{3}\right),
$$

and equation (16) is reduced to two polynomial equations $F=G=0$ for three unknowns $a_{1}=2 t, a_{2}=x, a_{3}=y$. We employed WMaple to check that

$$
\begin{aligned}
& 6 y^{2}-x^{3}+6 y x(1-2 t)+x^{2}\left(9 t^{2}-3 t-11\right)+x\left(37 t^{2}-19-12 t-15 t^{4}+6 t^{3}\right)+18 y-14 y t \\
& +12 t^{3} y-6 y t^{2}+28 t^{2}-26 t^{4}+3 t+7 t^{6}-3 t^{5}-9+9 t^{3}=0, \\
& a_{1}=2 t, \quad a_{2}=x, \quad a_{3}=y \\
& 2 y^{2} t^{2}-8 y^{2} x+126 x y t^{2}+15 t^{4} x^{2}-7 t^{6} x+90 x y t-14 t^{3} x y-105 t^{3} x^{2}+x^{4}+210 x^{2} t \\
& -75 t^{3} y+180 x+21 x^{3} t-525 t^{3} x-441 y+13 y x^{2} t-105 t^{4} y-21 y x^{2}-9 x^{3} t^{2}+125 t^{4} x \\
& +441 x t+118 x^{2}-270 t^{2}+315 y t^{2}-210 x y-100 x^{2} t^{2}-42 y^{2} t-60 y^{2}+t^{5} y+20 x^{3}+17 y t \\
& +81-441 t^{3}+315 t^{5}+180 t^{4}-45 t^{6}-63 t^{7}+147 t^{5} x-289 x t^{2}=0 .
\end{aligned}
$$

We seek the solutions as polynomials w.r.t. $t$ of degree 2 and 3 , respectively,

$$
x=\alpha t^{2}+\alpha_{1} t+\alpha_{2}, y=\beta t^{3}+\beta_{1} t^{2}+\beta_{2} t+\beta_{3} \Rightarrow \alpha=1 .
$$

If one seek a solution to the system (see Appendix) as polynomials w.r.t. $t$ of degree 1 and 2 for some values of $t$, one succeeds to find an additional list of commuting operators of order 
4 and 6 . For some specific values of $t$, the solutions are reduced to Bessel function of integer order.

Finally, we let $a_{1}=2 t, a_{2}=c_{1} t+c_{2}, a_{3}=c_{3} t^{2}+c_{4} t+c_{5}$. As a result, we find

$$
c_{1}=-10, \quad c_{2}=-21, \quad c_{3}=0, \quad c_{4}=8, \quad c_{5}=20 .
$$

Solving the system, we find the following values of $t, a_{i}, b_{j}$ and associated polynomials $P(z)$ and $Q(z)$,

\begin{tabular}{|l|l|l|l||l|l|l|l||l|l|}
\hline No. & $t$ & $a_{1}$ & $a_{2}$ & $a_{3}$ & $b_{1}$ & $b_{2}$ & $b_{3}$ & $b_{4}$ & $b_{5}$ \\
\hline \hline 1 & -2 & -4 & -1 & 4 & -3 & -8 & 12 & 16 & 0 \\
\hline \hline 2 & -3 & -6 & 9 & -4 & -6 & 7 & 6 & -8 & 0 \\
\hline \hline 3 & -4 & -8 & 19 & -12 & -9 & 25 & -15 & -26 & 24 \\
\hline \hline 4 & -1 & -2 & -11 & 12 & 0 & -20 & 0 & 64 & 0 \\
\hline \hline 5 & -5 & -10 & 29 & -20 & -12 & 46 & -48 & -47 & 60 \\
\hline \hline 6 & -6 & -12 & 39 & -28 & -15 & 70 & -90 & -71 & 105 \\
\hline
\end{tabular}

\begin{tabular}{|l|l|l|}
\hline No. & $P(z)$ & $Q(z)$ \\
\hline \hline 1 & $z(z-1)(z-4)(z+1)$ & $z^{2}(z-2)(z-4)(z+2)(z+1)$ \\
\hline \hline 2 & $z(z-4)(z-1)^{2}$ & $z^{2}(z-1)(z-2)(z-4)(z+1)$ \\
\hline \hline 3 & $z(z-1)(z-3)(z-4)$ & $z(z-1)(z-2)(z-3)(z-4)(z+1)$ \\
\hline \hline 4 & $z(z-1)(z+3)(z-4)$ & $z^{2}(z+4)(z-2)(z+2)(z-4)$ \\
\hline \hline 5 & $z(z-5)(z-1)(z-4)$ & $z(z-1)(z-3)(z-4)(z-5)(z+1)$ \\
\hline \hline 6 & $z(z-1)(z-7)(z-4)$ & $z(z-5)(z-1)(z-7)(z-3)(z+1)$ \\
\hline
\end{tabular}

Passing to operators (operators obtained by shift of root are regarded as equivalent), we obtain the following list,

$$
\begin{array}{ll}
e^{4 t} D(D+6)(D+8)(D+14), & e^{6 t} D(D+4)(D+8)^{2}(D+12)(D+16), \\
e^{4 t} D(D+6)(D+8)(D+10), & e^{6 t} D(D+4)(D+8)^{2}(D+10)(D+12), \\
e^{4 t} D(D+6)^{2}(D+8), & e^{6 t} D(D+4)(D+6)(D+8)^{2}(D+10), \\
e^{4 t} D(D+2)(D+6)(D+8), & e^{6 t} D(D+2)(D+4)(D+6)(D+8)(D+10), \\
e^{4 t} D(D+2)(D+8)(D+10), & e^{6 t} D(D+2)(D+4)(D+8)(D+10)(D+12), \\
e^{4 t} D(D+6)(D+12)(D+14), & e^{6 t} D(D+4)(D+8)(D+12)(D+14)(D+16) .
\end{array}
$$

\section{DIXMIER OPERATOR PAIR}

In addition to interesting generalizations of Dixmier example [2] constructed in works [5], [4], we consider briefly the issue on a role of the free parameter involved in all these examples. Bearing in mind the general formula

$$
\left[A^{n}, B\right]=A^{n-1} C+A^{n-2} C A+A^{n-3} C A^{2}+\cdots+C A^{n-1}, \quad C \stackrel{\text { def }}{=}[A, B],
$$

in the case of operators of orders 4 and 6 we let

$$
\begin{gathered}
A=A_{0}^{2}+a(x), \quad B=A_{0}^{3}+b(x) \circ A_{0}+A_{0} \circ b(x), \quad A_{0}=D^{2}+u(x), \\
A_{\lambda}=A+2 \lambda A_{0}+\lambda^{2}, \quad B_{\lambda}=B+3 \lambda^{2} A_{0}+\lambda\left(3 A_{0}^{2}+2 b\right)+\lambda^{3} .
\end{gathered}
$$

Then

$$
\left[B_{\lambda}, A_{\lambda}\right]=[B, A]+\lambda^{2}\left(3\left[A_{0}, A\right]+4\left[b, A_{0}\right]\right)+2 \lambda\left[B, A_{0}\right]+\lambda\left[3 A_{0}^{2}+2 b, A\right]=0 .
$$


Hence, if $4 b=3 a$, it follows from equation $[B, A]=0$ that $\left[B_{\lambda}, A_{\lambda}\right]=0$.

Under the condition $4 b=3 a$, the operator equation $[B, A]=0$ allows us to determine the functions $u(x)$ and $a(x)$. Indeed,

$$
4[B, A]=A_{0}^{2} A_{1}+A_{1} A_{0}^{2}-2 A_{0} A_{1} A_{0}+3\left(a \circ A_{1}+A_{1} \circ a\right), \quad A_{1}=\left[A_{0}, a\right]=2 a^{\prime} D+a^{\prime \prime} .
$$

Here we evaluate the coefficients at various powers of $D$. The coefficients at $D^{5}$ and $D^{4}$ cancel out thanks to the condition $4 b=3 a$, and the restriction for the coefficient at $D^{3}$ to vanish implies the equation $a^{\prime \prime \prime}=0$. At that, the coefficient at $D^{2}$ vanishes, and the coefficient at $D$ and the free term yield

$$
\begin{gathered}
3\left(a \circ A_{1}+A_{1} \circ a\right)=4\left(3 a^{\prime \prime} u^{\prime}+a^{\prime} u^{\prime \prime}\right) D+2\left(4 a^{\prime \prime} u^{\prime \prime}+a^{\prime} u^{\prime \prime \prime}\right) \quad \text { or } \\
3 a^{\prime \prime} u^{\prime}+a^{\prime} u^{\prime \prime}=3 a a^{\prime}, \quad a(x)=\left\{\begin{array}{l}
\alpha x^{2}+\gamma, \\
\beta x
\end{array} .\right.
\end{gathered}
$$

The solutions to the equation $3 a^{\prime \prime} u^{\prime}+a^{\prime} u^{\prime \prime}=3 a a^{\prime}$ are (cf. [5])

$$
u(x)=\frac{1}{2}\left\{\begin{array}{l}
\frac{1}{4} \alpha x^{4}+\frac{3}{4} \gamma x^{2}-\frac{C_{1}}{x^{2}}+C_{2}, \\
\beta x^{3}+C_{1} x+C_{2}
\end{array}\right.
$$

\section{APPENDIX}

The system of algebraic equations for the coefficients $a_{i}$ of the polynomial $P(z)$ in $(17)$ is

$$
\left\{\begin{array}{l}
2688 a_{1} a_{3}^{2}-1344 a_{1} a_{2}^{3}-588 a_{2} a_{1}^{5}+840 a_{3} a_{1}^{4}+1680 a_{1}^{3} a_{2}^{2}+2688 a_{3} a_{2}^{2} \\
+56448 a_{3}-28224 a_{1} a_{2}++26880 a_{3} a_{2}+63 a_{1}^{7}-10080 a_{3} a_{1}^{2}+8400 a_{2} a_{1}^{3} \\
-13440 a_{1} a_{2}^{2}-1260 a_{1}^{5}-4032 a_{3} a_{1}^{2} a_{2}+7056 a_{1}^{3}=0 \\
-31104+8064 a_{1} a_{3}^{2}-4032 a_{1} a_{2}^{3}-1764 a_{2} a_{1}^{5}+2520 a_{3} a_{1}^{4}+5040 a_{1}^{3} a_{2}^{2}+8064 a_{3} a_{2}^{2} \\
+42 a_{2} a_{1}^{6}+3072 a_{2} a_{3}^{2}+270 a_{1}^{6}+25920 a_{1}^{2}-2496 a_{3} a_{1} a_{2}^{2}-3264 a_{3} a_{1}+23040 a_{3}^{2} \\
-69120 a_{2}+169344 a_{3}-360 a_{2}^{2} a_{1}^{4}++864 a_{1}^{2} a_{2}^{3}-84672 a_{1} a_{2}+80640 a_{3} a_{2}-4320 a_{1}^{4} \\
+189 a_{1}^{7}-30240 a_{3} a_{1}^{2}+25200 a_{2} a_{1}^{3}-40320 a_{1} a_{2}^{2}-3780 a_{1}^{5}--7680 a_{2}^{3}+27744 a_{2} a_{1}^{2} \\
-3000 a_{2} a_{1}^{4}+3600 a_{3} a_{1}^{3}+9600 a_{1}^{2} a_{2}^{2}-17280 a_{3} a_{1} a_{2}-12096 a_{3} a_{1}^{2} a_{2}-45312 a_{2}^{2} \\
+672 a_{2} a_{3} a_{1}^{3}-192 a_{3}^{2} a_{1}^{2}-12 a_{3} a_{1}^{5}-384 a_{2}^{4}+21168 a_{1}^{3}=0, \\
\\
-46656+9024 a_{1} a_{3}^{2}-2592 a_{1} a_{2}^{3}-1674 a_{2} a_{1}^{5}+2280 a_{3} a_{1}^{4}+4320 a_{1}^{3} a_{2}^{2}+81344 a_{3} a_{2}^{2} \\
+63 a_{2} a_{1}^{6}+4608 a_{2} a_{3}^{2}++405 a_{1}^{6}+38880 a_{1}^{2}-3744 a_{3} a_{1} a_{2}^{2}-4896 a_{3} a_{1}+34560 a_{3}^{2} \\
-7776 a_{1}-103680 a_{2}+91584 a_{3}+288 a_{3}^{2} a_{1}^{3}++21 a_{3} a_{1}^{6}-192 a_{3} a_{2}^{3}-540 a_{2}^{2} a_{1}^{4} \\
+1296 a_{1}^{2} a_{2}^{3}-63072 a_{1} a_{2}+37824 a_{3} a_{2}-6480 a_{1}^{4}+189 a_{1}^{7}+432 a_{3} a_{1}^{2} a_{2}^{2}-1152 a_{3}^{2} a_{1} a_{2} \\
-18528 a_{3} a_{1}^{2}+20520 a_{2} a_{1}^{3}-30240 a_{1} a_{2}^{2}-3294 a_{1}^{5}-11520 a_{2}^{3}+41616 a_{2} a_{1}^{2}-4500 a_{2} a_{1}^{4} \\
+5400 a_{3} a_{1}^{3}+14400 a_{1}^{2} a_{2}^{2}+1152 a_{3}^{3}-25920 a_{3} a_{1} a_{2}-9456 a_{3} a_{1}^{2} a_{2}-180 a_{3} a_{2} a_{1}^{4} \\
-67968 a_{2}^{2}+1008 a_{2} a_{3} a_{1}^{3}-288 a_{3}^{2} a_{1}^{2}-18 a_{3} a_{1}^{5}-576 a_{2}^{4}+17928 a_{1}^{3}=0 .
\end{array}\right.
$$




\section{BIBLIOGRAPHY}

1. J.L. Burchnall, T.W. Chaundy. Commutative ordinary diff. operators, II. The identity $P^{n}=Q^{m}$ // Proc. Roy. Soc. London, ser. A. 1932. V. 134, No. 824. P. 471-485.

2. J. Dixmier. Sur les algebres de Weyl // Bull. Soc. Math. France. 1968. V. 96. P. 209-242.

3. A.B. Shabat. Lecture notes on soliton theory. Karachay-Cherkess State University, Karachaevsk, 2008. 60 pp.

4. O.I. Mokhov. Commuting ordinary differential operators of arbitrary genus and arbitrary rank with polynomial coefficients // Preprint. arXiv: 1303.4263.

5. A.E. Mironov. On commuting differential operators of rank 2// Sibir. electron. matem. izv. 2009. V. 6. P. 533-536. (in Russian).

6. A.B. Shabat, Z.S. Elkanova. Commuting differential operators // Teor. matem. fiz. 2010. V. 162, No. 3. P. 334-344. [Theor. Math. Phys. 2010. V. 162, No. 3. P. 276-285.]

7. A.B. Shabat, Z.S. Elkanova. Commuting differential operators in two-dimension // Ufimskij matem. zhur. 2011. V. 3, No. 2. P. 91-99. [ Ufa Math. J. 2011. V. 3, No. 2. P. 89-95.]

8. A.B. Shabat, Z.S. Elkanova, A.B. Urusova. Two-sided Darboux transformations // Teor. matem. fiz. 2012. V. 173, No. 2. P. 207-218. [Theor. Math. Phys. 2012. V. 173, No. 2. P. 1507-1517.]

Fatima Khasanovna Baichorova, PhD student, Aliyev Karachay-Cherkess State University,

Lenin str., 29

4369202, Karachaevsk, Karachay-Cherkess Republic, Russia

E-mail: fatima-kchgu@yandex.ru

Zariyat Saidakhmatovna Elkanova, PhD student, Aliyev Karachay-Cherkess State University,

Lenin str., 29

4369202, Karachaevsk, Karachay-Cherkess Republic, Russia

E-mail: z.109@mail.ru 